\title{
Non-alcoholic fatty liver disease and coronary atherosclerosis-does myocardial glucose metabolism provide the missing link?
}

\author{
S. M. Clifford, MD, FFRRCSI, ${ }^{a}$ and D. J. Murphy, MD, FRCR, FFRRCSI ${ }^{a}$ \\ a Department of Radiology, St Vincent's University Hospital, Dublin 4, Ireland
}

Received May 31, 2019; accepted Jun 3, 2019

doi: $10.1007 /$ s12350-019-01783-z

\section{See related article, pp. 610-620}

Non-alcoholic fatty liver disease (NAFLD) has become the most common cause of chronic liver disease in the United States, ${ }^{1}$ and is now a serious global public health concern, estimated to affect as many as $25 \%$ of the world's population. ${ }^{2}$ Characterized by the accumulation of excess triglycerides in hepatocytes, NAFLD is often asymptomatic initially, but can progress causing steatohepatitis, hepatic fibrosis and ultimately cirrhosis. The direct cause of NAFLD is uncertain, but its increasing prevalence has closely mirrored rising obesity levels in the western world, and it appears closely related to the metabolic syndrome. ${ }^{3-5}$ Aside from liver disease, patients with NAFLD are at increased risk of both fatal and nonfatal cardiovascular disease events. ${ }^{6,7}$ Indeed amongst patients with NAFLD, cardiovascular disease is a major cause of mortality, ${ }^{8}$ and has surpassed liver-associated complications as the leading cause of death. ${ }^{9}$ Despite this apparent strong link between NAFLD and cardiovascular disease, a definite cause and effect relationship has been difficult to firmly establish. ${ }^{4,10}$

In this issue of Journal of Nuclear Cardiology, Tang et al. ${ }^{11}$ explore the relationship between myocardial glucose metabolism and the development of coronary artery atherosclerosis in patients with NAFLD. The authors performed a retrospective analysis of 418 consecutive

Editorial comment on 'Non-alcoholic fatty liver disease with low myocardial glucose uptake is associated with coronary atherosclerosis' Tang et al. JNC 2019.

Reprint requests: D. J. Murphy, MD, FRCR, FFRRCSI, Department of Radiology, St Vincent's University Hospital, Elm Park, Dublin 4, Ireland; david.murphy@svhg.ie

J Nucl Cardiol 2021;28:621-3.

1071-3581/\$34.00

Copyright (C) 2019 American Society of Nuclear Cardiology. patients who underwent both whole body 2-deoxy-2$\left[{ }^{18} \mathrm{~F}\right]$ fluoro-D-glucose (FDG) positron emission tomography/computed tomography (PET/CT) and dedicated coronary computed tomography angiography (CCTA), for health or cancer screening or suspected coronary artery disease. Patients with a history of significant alcohol consumption or a history of liver or heart disease were excluded . CCTA images were assessed for the presence of plaque, plaque type (calcified, non-calcified or mixed plaque) and maximal coronary luminal stenosis; obstructive disease was defined as a luminal stenosis of $>50 \%$. Fatty liver was assessed for on the unenhanced attenuation correction CT component of the FDG PET/CT scans, by comparing mean hepatic and splenic attenuation; fatty liver was defined as a mean liver to spleen $\mathrm{HU}$ ratio of less than 1.0. FDG PET/CT images were analysed for semi-quantitative metabolic parameters including maximum standardized uptake value $\left(\mathrm{SUV}_{\max }\right)$ of the whole myocardium and mean liver SUV. To help benchmark myocardial glucose uptake with an internal control, the ratio of myocardial $\mathrm{SUV}_{\max }$ to hepatic $\mathrm{SUV}_{\text {mean }}$ was calculated (SUVratio).

Of the 418 subjects, the prevalence of NAFLD was approximately $19 \%(\mathrm{n}=80)$. On CCTA, patients with NAFLD had significantly higher rate of significant coronary artery stenosis than those without $(47.5 \%$ vs $14.8 \%$, $P<0.001)$. Subjects with NAFLD had significantly lower myocardial $\mathrm{SUV}_{\max }$ and ratio of myocardial $\mathrm{SUV}_{\text {max }}$ : hepatic $\mathrm{SUV}_{\text {mean }}$ (SUVratio) than those without NAFLD. There was a significantly higher proportion of low SUVratio in patients with NAFLD when compared to those without NAFLD (45.0\% vs $19.8 \%, P<0.001)$, and the authors found a significant negative correlation between myocardial FDG uptake and hepatic steatosis. The myocardial-to-hepatic SUV ratio values of individuals with NAFLD were divided into quartiles, and patients in the lower SUV ratio quartiles had a higher proportion of non-calcified coronary artery plaque than those in the higher quartiles. The association trend analysis revealed a 
strongly inverse correlation between myocardial FDG uptake and significant stenosis caused by non-calcified plaque $(P=0.001)$. On multivariate regression analysis after adjusting for clinically important variables, low myocardial-to-hepatic SUVratio was independently associated with the presence of non-calcified plaque and significant coronary artery stenosis.

These results suggest that individuals with NAFLD and reduced semi-quantitative myocardial FDG uptake have a higher prevalence of significant coronary artery stenosis, particularly secondary to non-calcified plaque. This finding has potential clinical significance, as these patients with NAFLD and reduced myocardial glucose metabolism might be at increased risk of future cardiovascular events, and could potentially benefit from more aggressive cardiovascular risk modification. Previous studies have demonstrated an association between NAFLD and coronary artery disease in terms of the presence of plaque, plaque volume, morphology and the presence of significant coronary artery stenosis. ${ }^{12,13}$ This higher risk of cardiovascular disease for patients with NAFLD may be somewhat explained by the greater prevalence of risk factors, such as diabetes, obesity, smoking and the metabolic syndrome, ${ }^{14}$ but this appears to only partly explain the link; the exact pathogenesis of the relationship between NAFLD and cardiovascular disease has yet to be mapped out. Insulin resistance and increased systemic inflammation have been proposed as possible mechanisms of this link, ${ }^{10}$ and the results of Tang et al. ${ }^{11}$ suggest that myocardial glucose metabolism may also play a role in the development of atherosclerosis in these individuals.

The association between NAFLD and cardiovascular disease also appears to go beyond coronary artery disease. In their analysis of the multi-ethnic study of atherosclerosis cohort, Shah et al. ${ }^{15}$ did not find an association between the presence of hepatic fat and overall cardiovascular disease outcomes, but they did show that individuals with NAFLD were more likely to have adverse features of left ventricular remodeling. Recent research by Vita et al. ${ }^{16}$ examined patients with NAFLD without evidence of coronary artery disease who underwent myocardial perfusion PET/CT. They observed that coronary microvascular dysfunction was more prevalent in patients with NAFLD, a known independent risk factor for major adverse cardiac events. A previous study by Tang et al. ${ }^{17}$ demonstrated that individuals with NAFLD and decreased myocardial glucose uptake on FDG PET were at higher risk of left ventricular diastolic dysfunction. The observation in this study by Tang et al. ${ }^{11}$ that reduced myocardial glucose metabolism may mediate the cardiovascular manifestations of NALFD is an interesting addition to the growing body of research regarding NAFLD and cardiovascular disease, and is of interest as we further explore and improving our understanding of the complex interaction between the heart and liver.

Certain limitations of this study by Tang et al. ${ }^{11}$ should be acknowledged. Firstly, the retrospective crosssectional study design limits the ability to determine a definite causal relationship between uptake of myocardial glucose metabolism in patients with NAFLD and the development of coronary atherosclerosis. The study population was exclusively Asian, and further studies will be required to see if these observations can be extrapolated to other population groups. The lack of any clinical follow up for cardiovascular disease events in the cohort is a limitation; it is not possible therefore to evaluate any potential association between NAFLD, reduced myocardial glucose uptake and future cardiovascular events.

Measuring myocardial glucose metabolism with FDG PET is fraught with difficulty owing to the different factors which govern myocyte glucose metabolism, principally diet and the fasting state. Variations in individual patient dietary preparation is therefore a potential confounder that may affect myocardial glucose metabolism measurement by FDG PET in this study. Although all patients in the cohort were required to fast for 6 hours prior to the FDG PET scans (standard oncology FDG PET/CT preparation), there were no other special dietary preparation methods used. We know from the FDG PET myocardial inflammation literature that diet on the day prior to FDG PET scanning affects myocardial glucose metabolism, with a high-fat, low-carbohydrate diet, along with a prolonged 12 to 18 hour fast causing significant reduction of physiological myocyte glucose metabolism in favour of free fatty acids. ${ }^{18,19}$ The degree to which individual patient dietary habits and length of fast may influence the results of Tang et al. ${ }^{11}$ is difficult to quantify, particularly given the retrospective study design, and is a potential avenue for further research in prospective studies.

The diagnosis of fatty liver in this study was based on analysis of the unenhanced CT attenuation correction scan component of the PET/CTs, and is a potential limitation. Although this is a validated method of NAFLD diagnosis, liver biopsy remains the gold standard. In terms of the non-invasive imaging diagnosis of fatty liver, MRI and particularly MR spectroscopy allows for a more sophisticated quantification of the severity of hepatic steatosis. ${ }^{20}$ This ability to quantify the extent and severity of hepatic steatosis non-invasively opens up further research opportunities-it would be very interesting to assess whether the individuals with NAFLD and reduced myocardial FDG uptake had more severe hepatic fat accumulation than the NAFLD patients with higher myocardial FDG uptake. We know that hepatic fat accumulation in NAFLD does not occur 
in isolation given its link with dietary habits and obesity, and certain further relevant risk factors could also be included in future analyses, such as visceral adipose tissue and subcutaneous adipose tissue measurements.

In terms of coronary artery plaque assessment on CTCA, Tang et al. ${ }^{11}$ characterized plaque type and stenosis, finding that individuals with NAFLD and reduced myocardial FDG uptake were more likely to have non-calcified plaque causing significant stenosis. This is in itself a significant observation- it would also be interesting to take the coronary plaque analysis further by examining for the presence of high risk coronary plaque features (for example spotty calcification, a necrotic core and the napkin ring sign), and any association with myocardial glucose uptake in NAFLD.

In conclusion, the results of this interesting study by Tang et al. ${ }^{11}$ suggest that the presence of reduced myocardial glucose metabolism as measured by FDG PET/CT in patients with NAFLD is associated with the presence of obstructive coronary artery stenosis, particularly secondary to non-calcified plaque. This association, however, is still cross-sectional, and may be the result of shared risk factors and pathways rather than definite cause and effect. Whilst the exact mechanism of the link between NAFLD and cardiovascular disease remains unclear, what this study highlights is the need to explore the potential role of altered myocardial glucose metabolism in the development of cardiovascular disease in patients with NAFLD in a large, prospective study with rigorous assessment of cardiovascular disease outcomes.

\section{Disclosure}

We have no conflicts of issue to declare.

\section{References}

1. Pickhardt PJ, Hahn L, Muñoz del Rio A, et al. Natural history of hepatic steatosis: Observed outcomes for subsequent liver and cardiovascular complications. AJR Am J Roentgenol 2014;202:752-8. https://doi.org/10.2214/AJR.13.11367.

2. Younossi ZM, Koenig AB, Abdelatif D, et al. Global epidemiology of nonalcoholic fatty liver disease-meta-analytic assessment of prevalence, incidence, and outcomes. Hepatology 2016;64:7384. https://doi.org/10.1002/hep.28524/full.

3. Mikolasevic I, Milic S, Turk Wensveen T, et al. Nonalcoholic fatty liver disease-A multisystem disease? WJG 2016;22:9488505. https://doi.org/10.3748/wjg.v22.i43.9488.

4. Byrne CD, Targher G. NAFLD: A multisystem disease. J Hepatol 2015;62:S47-64. https://doi.org/10.1016/j.jhep.2014.12.012.

5. Fargion S, Porzio M, Fracanzani AL. Nonalcoholic fatty liver disease and vascular disease: State-of-the-art. WJG 2014;20:13306-24. https://doi.org/10.3748/wjg.v20.i37.13306.

6. Targher G, Byrne CD, Lonardo A, et al. Non-alcoholic fatty liver disease and risk of incident cardiovascular disease: A meta- analysis. J Hepatol 2016;65:589-600. https://doi.org/10.1016/j.jhe p.2016.05.013.

7. Wu S, Wu F, Ding Y, et al. Association of non-alcoholic fatty liver disease with major adverse cardiovascular events: A systematic review and meta-analysis. Sci Rep 2016;6:33386. https://doi.org/ 10.1038/srep33386

8. Chalasani N, Younossi Z, Lavine JE, et al. The diagnosis and management of nonalcoholic fatty liver disease: Practice guidance from the American Association for the Study of Liver Diseases. Hepatology 2017;67:328-57. https://doi.org/10.1002/hep.29367.

9. Ong JP, Pitts A, Younossi ZM. Increased overall mortality and liver-related mortality in non-alcoholic fatty liver disease. J Hepatol 2008;49:608-12. https://doi.org/10.1016/j.jhep.2008.06. 018.

10. Adams LA, Anstee QM, Tilg H, Targher G. Non-alcoholic fatty liver disease and its relationship with cardiovascular disease and other extrahepatic diseases. Gut 2017;66:1138-53. https://doi.org/ 10.1136/gutjnl-2017-313884.

11. Tang K, Lin J, Ji X, et al. Non-alcoholic fatty liver disease with reduced myocardial FDG uptake is associated with coronary atherosclerosis. J Nucl Cardiol 2019;64:11-73. https://doi.org/10. 1007/s12350-019-01736-6.

12. Lee SB, Park G-M, Lee J-Y, et al. Association between non-alcoholic fatty liver disease and subclinical coronary atherosclerosis: An observational cohort study. J Hepatol 2018;68:1018-24. http s://doi.org/10.1016/j.jhep.2017.12.012.

13. Park HE, Kwak M-S, Kim D, et al. Nonalcoholic fatty liver disease is associated with coronary artery calcification development: A longitudinal study. J Clin Endocrinol Metab 2016;101:3134-43. https://doi.org/10.1210/jc.2016-1525.

14. Hamaguchi M, Kojima T, Takeda N, Nakagawa T. The metabolic syndrome as a predictor of nonalcoholic fatty liver disease. Ann Intern Med 2005;143:722-8.

15. Shah RV, Anderson A, Ding J, et al. Pericardial, but not hepatic, fat by $\mathrm{CT}$ is associated with $\mathrm{CV}$ outcomes and structure: The multi-ethnic study of atherosclerosis. JACC Cardiovasc Imaging 2017;10:1016-27. https://doi.org/10.1016/j.jcmg.2016.10.024.

16. Vita T, Murphy DJ, Osborne MT, et al. Association between nonalcoholic fatty liver disease at CT and coronary microvascular dysfunction at myocardial perfusion PET/CT. Radiology 2019. h ttps://doi.org/10.1148/radiol.2019181793.

17. Tang K, Zheng X, Lin J, et al. Association between non-alcoholic fatty liver disease and myocardial glucose uptake measured by 18F-fluorodeoxyglucose positron emission tomography. J Nucl Cardiol 2018. https://doi.org/10.1007/s12350-018-1446-x.

18. Kobayashi Y, Kumita S, Fukushima Y, et al. Significant suppression of myocardial 18F-fluorodeoxyglucose uptake using 24-h carbohydrate restriction and a low-carbohydrate, high-fat diet. J Cardiol 2013;62:314-9. https://doi.org/10.1016/j.jjcc.201 3.05.004.

19. Osborne MT, Hulten EA, Murthy VL, et al. Patient preparation for cardiac fluorine-18 fluorodeoxyglucose positron emission tomography imaging of inflammation. J Nucl Cardiol 2017;24:86-99. h ttps://doi.org/10.1007/s12350-016-0502-7.

20. Dulai PS, Sirlin CB, Loomba R. MRI and MRE for non-invasive quantitative assessment of hepatic steatosis and fibrosis in NAFLD and NASH: Clinical trials to clinical practice. J Hepatol 2016;65:1006-16. https://doi.org/10.1016/j.jhep.2016.06.005.

Publisher's Note Springer Nature remains neutral with regard to jurisdictional claims in published maps and institutional affiliations. 